\title{
Medulloblastoma: Clinicopathological Correlates of SHH, WNT, and Non-WNT/SHH Molecular Subgroups Analysis and Prognostic Significance: Mono-Institutional Series
}

\author{
Bermal HASBAY ${ }^{1}$, Fazilet KAYASELCUK ${ }^{1}$, Halil Ibrahim SUNER², Faik SARIALIOGLU³ \\ ${ }^{1}$ Baskent University Faculty of Medicine, Department of Pathology, Adana, Turkey \\ ${ }^{2}$ Baskent University Faculty of Medicine, Department of Neurosurgery, Adana, Turkey \\ ${ }^{3}$ Baskent University Faculty of Medicine, Department of Paediatrics, Division of Pediatric Oncology, Adana, Turkey \\ Corresponding author: Bermal HASBAY bermalhasbay@hotmail.com
}

\section{ABSTRACT}

AIM: To reevaluate the medulloblastoma cases according to histomorphological and molecular features, and to investigate the relationship between the prognostic factors of the new WHO classification by applying Beta-catenin, YAP1, GAP1, p53, and INI1 antibodies immunohistochemically.

MATERIAL and METHODS: This study includes 41 patients who have been diagnosed with medulloblastoma between $2007-2019$ in Pathology Department. Immunohistochemically, P53, Beta-catenin, YAP1, GAP1, and INI1 immune markers were applied, and the relationship between the results and the prognostic parameters was evaluated statistically.

RESULTS: When 41 patients were classified into WHO medulloblastoma histological subtype groups according to histomorphological features, $22(53.7 \%)$ patients were classified as classical type, 11 (26.8\%) patients as desmoplastic nodular type, and 8 (19.5\%) patients as large cell/anaplastic type medulloblastoma.

According to their molecular characteristics, 14 (34.1\%) patients were in the Non-WNT/SHH group, 5 (12.2\%) patients were SHH mutant, 17 (41.5\%) patients were SHH wild, and 5 (12.2\%) patients were in the WNT active group. There was no statistically significant correlation between age, gender, tumor size, recurrence, Ki67 proliferation index with molecular types and histopathological types.

CONCLUSION: In our study, metastasis at the time of diagnosis, histological large cell anaplastic type, immunohistochemical p53 positivity, molecular SHH mutant type were the statistically significant indicators of worse prognosis and shorter survival time.

KEYWORDS: Medulloblastoma, Histological subtype, Molecular characteristic, Immunohistochemistry

\section{INTRODUCTION}

$\mathrm{M}$ edulloblastomas $(\mathrm{MB})$ are high-grade, malignant central nervous system (CNS) tumors that comprise nearly $10 \%$ of all childhood CNS tumors (4).

Until the WHO 2016 classification, medulloblastomas were mostly classified histomorphologically. There were four main types (classic, desmoplastic/nodular, medulloblastoma with extensive nodularity, large-cell / anaplastic) in this classification. In recent years, increasing molecular pathology studies and the relationship between genetic anomalies, tumor types, and prognosis have led to a new molecular-based classification in MBs in addition to histomorphological classification. In the WHO 2016 CNS classification, medulloblastomas are classified into four genetic subtypes as well as histomorphological typing. These subtypes are WNT, SHH, Non-WNT/SHH groups. Non-WNT/SHH group is divided into Group 3 and Group $4(1,2,4,18,25)$. In the first studies, Medulloblastoma subgroup genes have been identified using the next-generation sequencing method (NGS) (17). Although NGS gives
Bermal HASBAY (1) : 0000-0002-7941-7962 Fazilet KAYASELCUK (10): 0000-0002-1180-3840
Halil Ibrahim SUNER (D) : 0000-0002-5957-8611

Faik SARIALIOGLU (D): 0000-0002-8257-810X 
much more precise results than the immunohistochemical (IHC) method, its use is limited since it is an expensive method. With advancing studies, the guideline to determine WNT active, SHH active, and Non-WNT/SHH groups using immune biomarkers was set (17).

Nearly all WNT active tumors are classic type medulloblastoma. WNT active tumors with an LC/A morphology, however, have been reported to be very rare. The WNT pathway activation can be demonstrated by the accumulation of beta-catenin immunoreactivity in tumor cell nuclei (4). $\mathrm{SHH}$-activated medulloblastomas express a signature of the activated hedgehog signals, and proteins such as GAB1 and SFRP1 have been observed in this pathway. Most Non-WNT/ SHH medulloblastomas have a classic morphology and are excluded from the WNT activated and SHH-activated groups by molecular testing (4).

Currently, the most practical method for determining the molecular subgroups of MBs is to use YAP1, GAP1, Betacatenin, and p53 immunohistochemical antibodies.

Of these antibodies, GAB1 indicates SHH activation, while YAP1 indicates SHH or WNT pathway $(1,4)$. The YAP1 gene is responsible for the regulation of the signal pathway involved in development, growth, repair, and homeostasis. YAP1 has been specifically shown in medulloblastomas regulated by SHH and WNT pathways.

GAB1 gene plays a role in cellular growth, transformation and apoptosis. GAB1 protein is particularly effective in medulloblastomas in the $\mathrm{SHH}$ group.

TP53, a well-known tumor suppressor gene, is also used in the molecular subtyping of MBs. As a result of TP53 mutation, cells divide uncontrollably and form tumors. The Tp53 mutation indicates a poor prognosis for SHH active medulloblastomas.

Beta-catenin acts as a regulator protein in the WNT signaling pathway (1). Beta-catenin can be expressed both nuclear and cytoplasmic. Cytoplasmic beta-catenin is active, but nuclear beta-catenin positivity indicates the activation of the WNT signaling pathway (1).

INI1 (SMARCB1) is expressed in the nuclei of all normal cells and can readily be identified by using immunohistochemistry (11). Monosomy 22 was described in a small series of atypical teratoid/rhabdoid tumors(ATRT) of the CNS. INI1 gene has been implicated as a tumor suppressor gene in ATRTs and peripheral rhabdoid tumors (9). Since the differential diagnosis of medulloblastoma from other embryonal tumors (ATRT, PNET, medulloepithelioma, etc.) should be made, routine antibodies should be added immunohistochemically at INI1.

The determination of molecular subtypes, as well as histopathological subtypes in pathology reports, will provide the clinician with more factual information for determining the disease's prognosis and treatment protocols. In this study, we reevaluated the medulloblastoma cases that are previously diagnosed and classified them based on their histomorphological features, and we immunohistochemically applied p53, Beta-catenin, YAP1, GAP1, and INI1 antibodies to determine the molecular subtypes of these cases in line with the current $\mathrm{WHO}$ classification and correlate the current dual classification with prognosis.

\section{MATERIAL and METHODS}

This study includes 41 patients diagnosed with medulloblastoma between 2007-2019 in Pathology Department who have sufficient tissue in their paraffin blocks for further evaluation and immunohistochemical studies.

Immunohistochemically, P53, Beta-catenin, YAP1, GAP1, and INI1 immune markers were applied to sections prepared from paraffin blocks, and the relationship between the results and the prognostic parameters were evaluated statistically.

Brand, dilutions, and clones for immunohistochemical studies are indicated (Table I). In terms of control tissue, normal colon and colon carcinoma for Tp53 and Beta-catenin, tonsil blocks for GAB1 and YAP1, and skin tissue for INI1 were used.

In the study, the positivity of Beta-catenin was evaluated as positive for nuclear staining in more than $5 \%$ of cells. Those who were cytoplasmic and/or membranous positive were considered negative. Cases showing nuclear beta-catenin expression were included in the group of WNT active tumors.

YAP1; $50 \%$ or more cytoplasmic or nuclear staining was evaluated as positive, and positive cases were included in the category of tumors of the WNT or SHH group (Figure 1).

GAB1; $50 \%$ and more cytoplasmic staining was evaluated as positive, and these cases were classified as $\mathrm{SHH}$ group (Figure 2).

In P53 staining, if more than $50 \%$ of the cells have strong nuclear staining, mutant and $1-50 \%$ staining was classified in the non-mutant (wild type) category.

Table I: Brand, Dilution and Clones of Immunohistochemical Antibodies

\begin{tabular}{|c|c|c|c|}
\hline Antibodies & Dilution & Brand & Clone \\
\hline Beta-catenin & ready to use & Dako & B-catenin -1 \\
\hline GAB-1 & $1 / 400$ & Santa Cruz Biotechnology & (c-5) sc-28322 \\
\hline YAP-1 & $1 / 125$ & Invitrogen & Yap-1 antibody \\
\hline P53 & $1 / 800$ & Leica & DO7 \\
\hline $\mathrm{INI}-1$ & $1 / 300$ & Cell Marque & MRQ-27 \\
\hline
\end{tabular}


Table II: Immunohistochemical Profile of Molecular Subtypes

\begin{tabular}{lcccc}
\hline & GAB1 & YAP1 & B-catenin & P53 \\
WNT active & Negative & $\begin{array}{c}\text { Nuclear or cytoplasmic } \\
(>\% 50)\end{array}$ & $>\% 5$ nuclear positive & $\begin{array}{c}\text { between 1-50\% positive } \\
\text { or negative }\end{array}$ \\
\hline SHH active & Cytoplasmic $(>50 \%)$ & Nuclear or cytoplasmic & $\begin{array}{c}\text { Cytoplasmic and } \\
\text { membranous negative }\end{array}$ & $\begin{array}{r}\text { between 1-50\% positive } \\
\text { or negative }\end{array}$ \\
\hline SHH mutant & Cytoplasmic $(>50 \%)$ & Nuclear or cytoplasmic & Cytoplasmic or & $>\% 50$ nuclear positive \\
Non-WNT/SHH & Negative & Negative & Negative & Negative \\
\hline
\end{tabular}

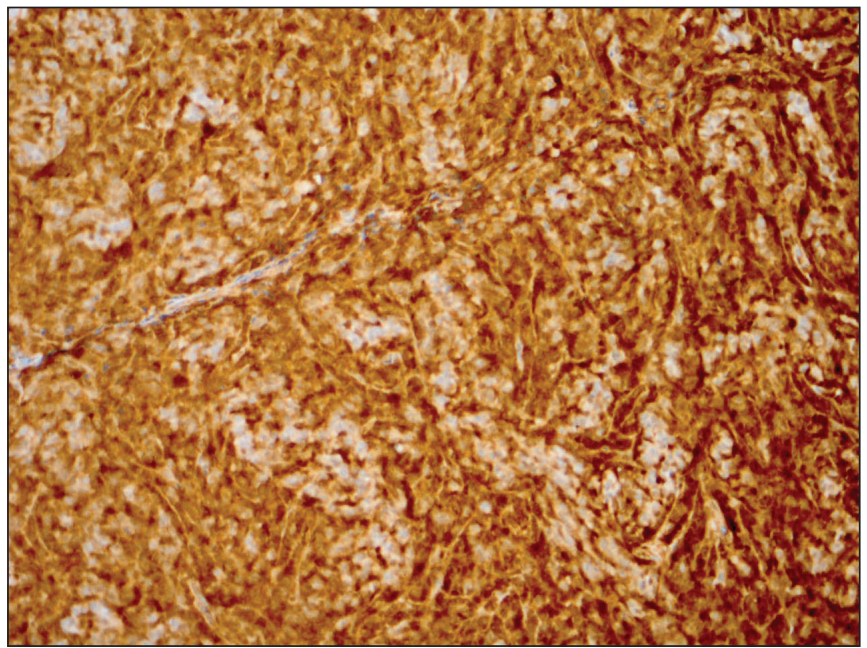

Figure 1: YAP1 positivity (IHC x200).

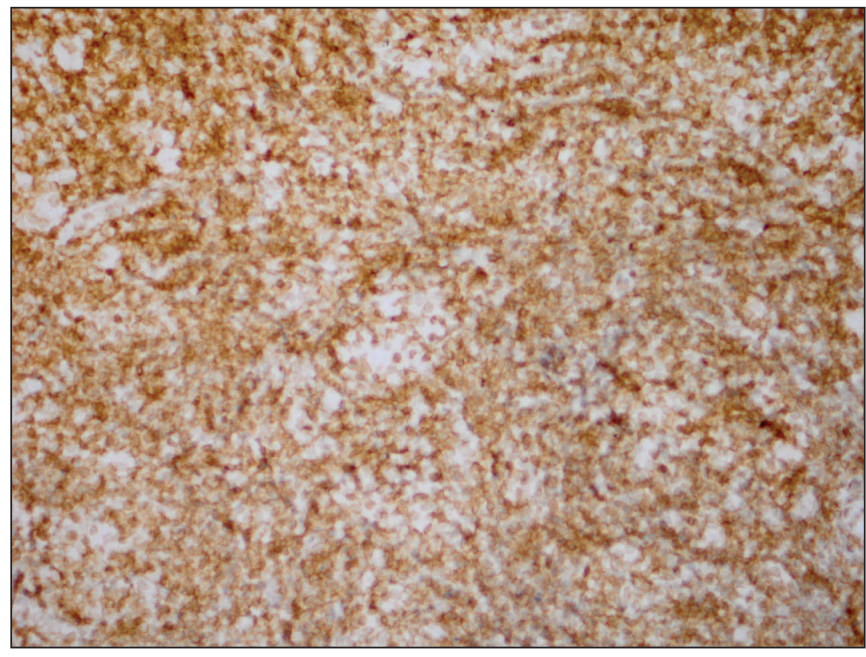

Figure 2: GAP1 cytoplasmic positivity (IHCx200).

For INI1, nuclear-stained cases were considered positive, and cases with nuclear expression loss were considered negative.

YAP1, GAP1, and Beta-catenin negative cases were included in the Non-WNT/SHH group. Since the immunohistochemical method alone is not sufficient for Group 3 and Group
4, this group was classified as Non-WNT/SHH. The immunohistochemical profile of the molecular subtypes is summarized (Table II).

Statistically, age, gender, histopathological type, follow-up duration, treatment methods were determined, and durations were noted as months.

Statistical analysis was performed using the statistical package SPSS software (Version 17.0, SPSS Inc., Chicago, IL, USA). All numerical data were expressed as median values (MinimumMaximum) or as proportions. The association with overall survival was analyzed using the log-rank test to examine their relationship when different variables were applied.

The survival curve was plotted using the standard KaplanMeier methodology. For all the statistical tests used, the error level was taken as 0.05 , and $P$ values less than 0.05 were considered significant.

Since this is a retrospective study, patients did not provide written informed consent for their clinical records to be used in this survey.

\section{RESULTS}

The ages of the patients range from 3 months to 25 years, and the average age is $9.5+/-6.01$. Twenty-one of the patients were female, and 20 were males. The most common symptoms were headache (56\%), vomiting /nausea (45\%), diplopia (4\%), and ataxia, which were associated with increased intracranial pressure and dysfunctional cerebellar symptoms. Duration of symptoms varied from 3 days to five months, and the median duration of symptoms was one month. The follow-up period of the patients ranged from 9 to 144 months, with an average of 64.5 months.

The number of cases with metastasis (or spinal spread) at the time of the diagnosis was 10. Recurrence was observed in 8 patients. Four of the patients with metastasis were girls, and six were boys. Five of the patients with recurrence were girls, and three were boys. Six of the metastatic patients have died during the follow-up, which is statistically significant $(p=$ 0.039). Six of the relapsed cases were CLS type, and 2 were LC/A.

Of the 41 patients in the study, 32 (78\%) were alive, while nine patients $(22 \%)$ have died during the follow-up. Of the 


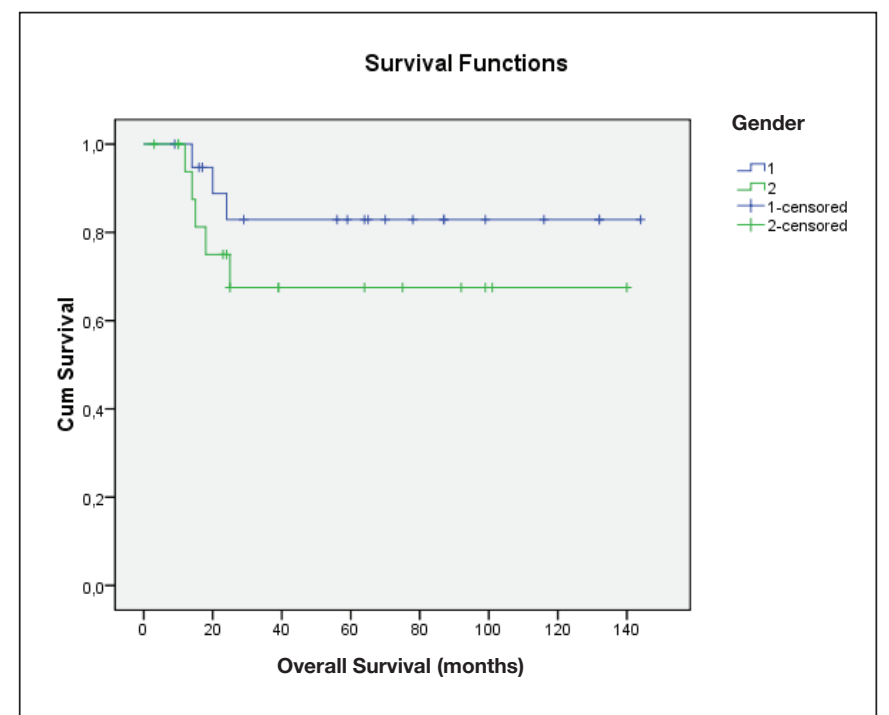

Figure 3: Relationship between gender and survival $(p=0.281)$.

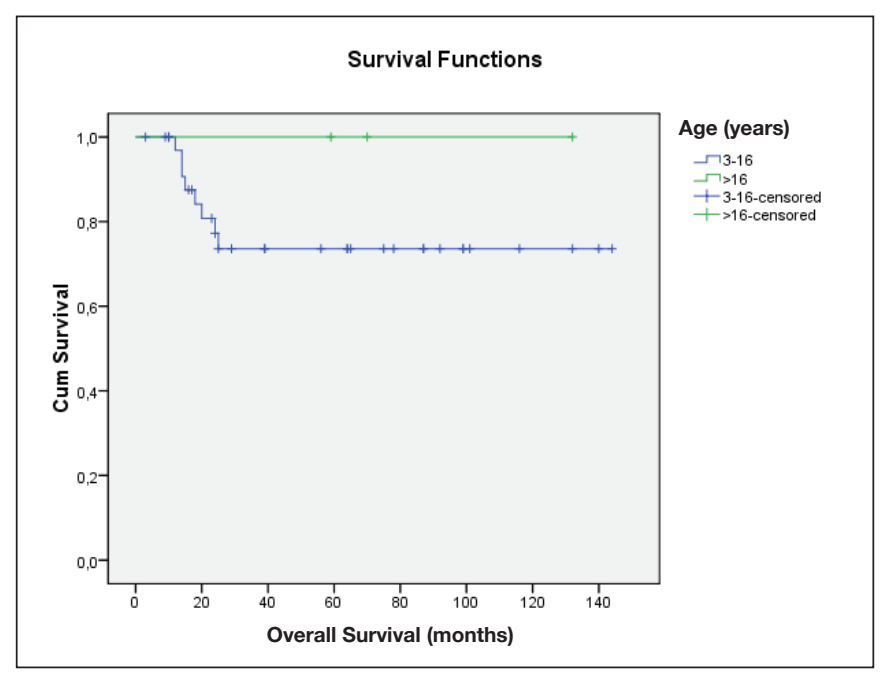

Figure 4: Relationship between age and survival $(p=0.341)$.

deceased patients, three were female, six were male, and their ages at the time of death ranged from 5 months to 16 years. Two of the deceased patients did not receive treatment at our center, and one case (5-months-old) died shortly after diagnosis without the opportunity to receive treatment. Seven of the deceased patients were in the $\mathrm{SHH}$ group, and two were in the Non-WNT/SHH group. Histopathologically, five were in the LC/A group, three were in the CLS group, and one was in the DNT group.

In terms of treatment, 33 patients received chemotherapy (CT) + Radiotherapy (RT), and five patients received RT only. Two patients did not receive treatment after being diagnosed at our center. Treatment could not be started because one patient was ex shortly after the diagnosis. In our hospital, the ICEICbE protocol is applied as chemotherapy. As an RT dose, a maximum of 54 gray is applied to the posterior fossa and a maximum of 45 gray to the craniospinal area.
When 41 patients were classified into the WHO medulloblastoma histological subtype groups based on histomorphological features, 22 (53.7\%) patients were classified as CLS type, $11(26.8 \%)$ patients as DNT type, and 8 (19.5\%) patients as LC/A type medulloblastoma. In our series, extensive nodular type medulloblastoma was not observed. There was no statistically significant correlation between age (Figure III), gender (Figure 4), tumor size, recurrence, Ki67 proliferation index, and histopathological types. Five of the deceased patients were LC/A type, and the death rate in this group was statistically significantly higher than other subtypes $(p=0.01)$.

The correlation between the average survival time and histopathological types was as follows: 52.85 months in LC/A, 114.17 months in DNT, and 123.21 months in CLS type. The correlation between the average survival and histopathological types was statistically significant according to the log-rank test, and the p-value was 0.037 . The estimated survival time of patients without metastasis was 137.81 months, while the estimated survival time of patients with metastasis was 60.12. The difference was statistically significant according to the log-rank test, and its p-value was 0.009. The estimated survival time was 50.80 months in p53 positive cases and 121.67 months in p53 negative cases, which was statistically significant $(p=0.023)$. In the follow-up of our patients, overall survival for five years was $90.5 \%$, seven years $75 \%$, and nine years $37 \%$.

According to their molecular characteristics, 14 (34.1\%) patients were in the Non-WNT/SHH group, 5 (12.2\%) patients were $\mathrm{SHH}$ mutant, 17 (41.5\%) patients were $\mathrm{SHH}$ wild, and $5(12.2 \%)$ patients were in the WNT active group. Seven of 9 deceased patients were in the $\mathrm{SHH}$ group, which was statistically significant $(p=0.036)$. In terms of metastasis, 8 (80\%) metastatic cases were $\mathrm{SHH}$-wild type, which was also statistically significant $(p=0.051)$. There was no significant difference between the molecularly classified groups and the survival time $(\mathrm{p}=0.114)$ (Table III). The Ki67 proliferation index ranged from $8 \%$ to $70 \%$ and was 27.5 on average.

There was no statistically significant correlation between age, gender, tumor size, recurrence, Ki67 proliferation index, and molecular types. When we look at the molecular subtype distribution of histological types, all of the DNTs were observed in the $\mathrm{SHH}$ group, which was compatible with the literature. The majority of classical medulloblastomas (54.5\%) were included in the Non-WNT/SHH group. LC/A type is mostly (57.1\%) SHH mutant type. WNT active tumors include classic type medulloblastomas; $\mathrm{SHH}$ active tumors were including all morphologic types. Non-WNT/SHH molecular type was including classic or LC/A type medulloblastomas (Table IV).

P53 was strongly positive in 5 patients and negative in 36 patients. Four of the p53 positive, five patients were large cell anaplastic type, and one was the classical type. While positive staining was observed in 39 patients with INI1, loss of staining was observed in two patients. One of the negative patients was WNT active-classical type, and the other was SHH mutant -LC/A. 
Table III: Statistical Results and p Values of Prognostic Parameters

\begin{tabular}{|c|c|c|c|c|c|c|c|c|}
\hline & \multirow{2}{*}{$\begin{array}{l}\text { Estimate } \\
\text { Mean }\end{array}$} & \multirow{2}{*}{ Std. Error } & \multicolumn{2}{|c|}{ 95\% Confidence Interval } & \multirow{2}{*}{$\begin{array}{c}1 . y e a r \\
\text { survivor } \%\end{array}$} & \multirow{2}{*}{$\begin{array}{c}\text { 3.year } \\
\text { survivor \% }\end{array}$} & \multirow{2}{*}{$\begin{array}{c}\text { 5.year } \\
\text { survivor \% }\end{array}$} & \multirow{2}{*}{$\mathbf{p}$} \\
\hline & & & Lower Bound & Upper Bound & & & & \\
\hline Overall Survival & 113,741 & 9,365 & 95,386 & 132,096 & 97.1 & 76.0 & 76.0 & - \\
\hline \multicolumn{9}{|l|}{ Tumor type } \\
\hline $\mathrm{LC} / \mathrm{A}$ & 52,857 & 15,804 & 21,881 & 83,833 & 71.4 & 42.9 & 42.9 & \multirow{3}{*}{0.037} \\
\hline DNT & 114,167 & 16,280 & 82,259 & 146,075 & 83.3 & 83.3 & 83.3 & \\
\hline CLS & 123,121 & 11,034 & 101,495 & 144,747 & 94.7 & 83.6 & 83.6 & \\
\hline \multicolumn{9}{|l|}{ Localization } \\
\hline $4^{\text {th }}$ ventricle & 57,750 & 5,846 & 46,291 & 69,209 & 87.5 & 87.5 & 87.5 & \multirow{2}{*}{0.460} \\
\hline Cerebellum & 108,497 & 11,423 & 86,109 & 130,885 & 96.2 & 71.7 & 71.7 & \\
\hline \multicolumn{9}{|l|}{ Gender } \\
\hline Female & 122,711 & 11,221 & 100,718 & 144,703 & 94.7 & 82.9 & 82.9 & \multirow{2}{*}{0.281} \\
\hline Male & 100,063 & 14,787 & 71,080 & 129,045 & 93.8 & 67.5 & 67.5 & \\
\hline \multicolumn{9}{|l|}{ Metastasis } \\
\hline Available & 137,810 & 6,041 & 125,969 & 149,650 & 95.2 & 95.2 & 95.2 & \multirow{2}{*}{0.009} \\
\hline Not available & 60,113 & 14,539 & 31,615 & 88,610 & 90.0 & 67.5 & 50.6 & \\
\hline \multicolumn{9}{|l|}{ Relapse } \\
\hline Available & 126,004 & 9,654 & 107,082 & 144,927 & 95.7 & 85.5 & 85.5 & \multirow{2}{*}{0.156} \\
\hline Not available & 94,750 & 20,673 & 54,231 & 135,269 & 87.5 & 62.5 & 85.5 & \\
\hline \multicolumn{9}{|l|}{ p53 } \\
\hline Negative & 121,666 & 9,089 & 103,850 & 139,481 & 96.7 & 82.2 & 82.2 & \multirow{2}{*}{0.023} \\
\hline Positive & 50,800 & 18,403 & 14,730 & 86,870 & 60.0 & 40.0 & 40.0 & \\
\hline \multicolumn{9}{|l|}{ Molecular type } \\
\hline Non WNT/SHH & & & & & 91.7 & 83.3 & 83.3 & \multirow{4}{*}{0.114} \\
\hline SHH mutant & & & & & 60.0 & 40.0 & 40.0 & \\
\hline SHH wild & & & & & 92.9 & 73.9 & 73.9 & \\
\hline WNT active & & & & & 100 & 100 & 100 & \\
\hline
\end{tabular}

CLS: Classical type medulloblastoma, DNT: Desmoplastic nodular type medulloblastoma, LC/A: Large cell/anaplastic type medulloblastoma.

Table IV: Distribution According to Molecular Subtypes of Histological Subtypes

\begin{tabular}{lccccc}
\hline & WNT active & $\begin{array}{c}\text { SHH active-p53 } \\
\text { Mutant }\end{array}$ & $\begin{array}{c}\text { SHH active-p53 } \\
\text { 'Wild ' type }\end{array}$ & $\begin{array}{c}\text { Non-WNT/Non- } \\
\text { SHH }\end{array}$ & Total \\
\hline CLS & 5 & 1 & 4 & 12 & 22 \\
\hline DNT & & 0 & 12 & 0 & 12 \\
\hline LC/A & 0 & 4 & 1 & 17 & 7 \\
\hline Total & 5 & 5 & 17 & 41 \\
\hline
\end{tabular}




\section{DISCUSSION}

Medulloblastomas are the most common brain tumors after pilocytic astrocytoma in childhood. The male/female ratio is around $1.5 / 1$. The most common clinical symptoms were headache, nausea/vomiting, and dizziness (4). In our study, the male/female ratio was around 1 , and the most common complaint was a headache. Most of the cases originate from the cerebellum midline or vermis in the posterior fossa $(1,4)$. Of the tumors in our series, 30 were located in the cerebellum and 11 in the $4^{\text {th }}$ ventricle.

In terms of prognosis, while tumor size was previously accepted as an important criterion, it was determined that it no longer had any effect. In current studies, age, metastasis status at the time of diagnosis, and genetic features have become more important. Patients who are older than three years, do not have metastases, and whose remnant tumor load is less than $1.5 \mathrm{~cm}^{3}$ after surgery are accepted in the standard-risk group. Because tumor biology is more aggressive in patients younger than three years of age and the tumor has a low chance of total resection. Close clinical follow-up and more aggressive treatment should be considered for these patients $(8,24)$.

Regardless of the histopathological type, there are publications showing the presence of metastasis at the time of diagnosis as the worst prognostic factor $(16,22) .82 .3 \%$ of our cases were between the ages of 3-16, and the number of patients aged under three years old was three. We had ten patients with metastasis at the time of diagnosis; six of them died during the follow-up, which was found to be statistically significant $(p=0.039)$. At the same time, the difference in estimated survival times between patients with and without metastases was evident, which was found to be statistically significant $(p=0.009)$.

Although it was previously believed that MBs originated from embryonic or immature cells, recent molecular studies have revealed that cell origin varies according to subgroups (15). It is accepted that WNT active MBs originate from the dorsal brainstem cells in the lower rhombic lip, and those with activate $\mathrm{SHH}$ originate from the external granular layer cells $(1,6)$.

Because the medulloblastoma histological subtypes are associated with the prognosis, it is important to specify the subtypes in the pathology reports. While the MBs in the DNT and MBEN subtypes show a better clinical prognosis, the prognosis is worse in LC/A and CLS type MBs. In our serial, LC/A type medulloblastomas were the ones with the worst prognosis and metastasis at the time of diagnosis, which was statistically significant $(\mathrm{p}=0.037)$.

LC/A type and SHH pathway active P53 mutant forms are more aggressive. In our cases, the LC/A type and P53 mutant forms had a significantly worse prognosis $(p=0.01)$.

WHO 2016 has classified medulloblastomas into four genetic subgroups: WNT, SHH, Non-WNT/SHH (group 3 and group 4 in itself) based on gene expression and methylation profiles. Immunohistochemically, the YAP1 positivity indicates the WNT or SHH pathway, while GAP1's cytoplasmic positivity indicates the $\mathrm{SHH}$ pathway. Nuclear positive staining in at least $10 \%$ of cells with beta-catenin indicates the WNT pathway. In the negativity of YAP1, GAP1, and Beta-catenin, the tumor is classified in the Non-WNT/SHH group $(4,5)$.

The most common group in the literature is the Non-WNT/ $\mathrm{SHH}$ group, and it is followed by the $\mathrm{SHH}$ active group and the WNT active group, respectively (5). In our serial, 14/41 (34.1\%) of the cases are in the Non-WNT/SHH group, while the SHH group was the most common. This can be explained by the presence of $29.3 \%$ of our cases being in the DNT group and the activity of the SHH pathway in the DNT group.

The WNT gene uses two types of pathways. The first one is the beta-catenin pathway, which is also important in tumorigenesis, and the other is the pathway that regulates intracellular calcium release $(5,7)$.

WNT pathway active tumors constitute $10-14 \%$ of all medulloblastomas. In this group, more $\mathrm{MBs}$ in classical histology and less LC/A morphology are observed $(5,12)$. In our serial, 5/41 (12.2\%) cases were grouped as WNT active, all of which are histopathologically grouped as the classic type. These results are consistent with the literature.

SHH pathway active tumors account for $30 \%$ of all medulloblastomas $(4,12)$. This group of tumors is divided into two groups as "p53 mutant" and "p53/wild type" according to the status of Tp53 mutation $(4,26)$. The number of active tumors in the SHH pathway in our serial was 22/41 (53.7\%). The number of SHH active-p53 mutant cases was 5, and the number of SHH active-p53 wild-type cases was 17. Twelve of the cases were DNT, 4 of them were CLS, and 1 was LC/A. All DNT medulloblastomas are $\mathrm{SHH}$ active tumors in our serial, and this finding is compatible with the literature $(1,2)$. The higher number of $\mathrm{SHH}$ groups in this study may be due to the high number of DNT medulloblastoma cases.

In P53 mutant MBs, classical, LC/A, and DNT morphology is observed. In the P53, wild type, classical, LC/A, DNT, and MBEN morphology is dominant (16). Within the WNT subgroup, most P53 mutant cases were of the classical medulloblastoma, but LC/A histology was commonly observed in the P53 mutant SHH cases (21). Unlike WNT active tumors, the P53 mutation shows a poor prognosis for $\mathrm{SHH}$ active medulloblastomas (26). Our number of P53 mutant cases was 5. One of the cases was classical, and four of them were in LC/Amorphologic group. These rates are compatible with the literature. The estimated survival time for p53 positive cases was 50.80 months and 121.67 months for negative cases, which was statistically significant $(p=0.023)$.

INI1 gene has been implicated as a tumor suppressor gene in ATRTs and peripheral rhabdoid tumors (9). ATRT is a highgrade embryonal tumor that responds poorly to conventional type therapy protocols. With recent studies, longer survival time is achieved with more intensive, aggressive therapy protocols. Therefore, differential diagnoses should be made, especially from other embryonal tumors (medulloblastoma, PNET, medulloepithelioma, etc.). The INI1should be immunohistochemically included in the panel. Haberler et al. (9). observed a $3.8 \%$ rate of INI1 negative medulloblastoma. In 
our serial, negativity was observed in $2 / 41$ (4.8\%) cases. In their study, they observed that patients who had negative INI1 had a worse prognosis. One of the two cases in our serial is one of our deceased patients. However, the number of our cases is limited for evaluating the relationship between INI1 negativity and prognosis.

The identification of the molecular subtypes also provides important information to the clinic in terms of treatment and prognosis. It has been discovered in recent studies that WNT active tumors have a better prognosis, and SUFU and SMO antagonists can be used to treat $\mathrm{SHH}$ active tumors (13). Prognosis in children is dependent on age, molecular subtype, and surgical resection. There was no statistically significant relationship between molecular subtype and age, gender, tumor size, and survival time in our study.

The treatment modalities are surgical resection, CT, and RT combinations. Surgery is considered as a standard part of treatment for histologic confirmation of tumor type $(2,20)$. The use of postoperative RT provides the greatest efficiency in medulloblastoma patients. The commonly used RT dose is 54 Gy to the posterior fossa and a standard dose of around 35 Gy for lesion-free craniospinal target $(10,19,25)$. Many patients experience hearing loss, secondary tumors, endocrine, vascular and urological problems due to treatment (especially secondary to RT). Some groups have a better prognosis and better surveys. Therefore, determining histological and molecular subgroups and accordingly organizing treatment protocols will prevent unnecessary treatment and related side effects. Our institution is a significant oncology center in its region. Patients operated in different centers are referred to our hospital either to confirm the pathological diagnosis and /or to receive chemo-radiotherapy. These patients have many clinical variables, including time intervals between initial diagnosis and admission to our hospital, previously received treatments, clinical status at the time of admission, and the preoperative and postoperative assessments. We have little to no information about some of these variables. Socioeconomic and cultural problems also cause difficulties in applying standard treatment protocols. It is difficult to standardize the outcomes of the treatments in patients of whom we have no control over the initial diagnostic and therapeutic processes. On the other hand, we have to assess the novelties in treatment and apply a precise treatment protocol. Molecular classification of medulloblastoma should be applied to all patients to practice new approaches in the treatment protocols. Histopathologic and molecular subclassification of MBs can modify therapeutic planning (23). Recent studies revealed that drugs such as SUFU and SMO antagonists could be used for treatment, especially for $\mathrm{SHH}$ active tumors $(3,13)$. The presence of targeted therapy that can suppress the WNT and SHH pathway can determine the future adjuvant therapeutic process. Novelties in the molecular classification may help us form standard treatment protocols by lowering the required doses- thus the adverse effects - of chemo and radiation therapies.

\section{CONCLUSION}

As a result, histological and molecular subtype distributions in our study were found to be compatible with the literature. While the CLS type and DNT follow-up times were very close to each other in prognostic terms, the LC/A type was determined as the worst group. In our study, metastasis at the time of diagnosis, histologicalLC/A type, immunohistochemicalp53 positivity, molecular $\mathrm{SHH}$ mutant (p53 positive) type were the statistically significant indicators of worse prognosis and shorter survival time. Studies showed that immunohistochemical p53 overexpression is a reliable marker indicating a mutation in the TP53 gene. In TP53, mutations, mutant p53 proteins are no longer "guardians of the genome," on the contrary, "guardians of the tumor cells," and the adaptive stress processes orchestrated by mutant p53 to protect tumor cells causes a positive loop of mutant p53 production and its accumulation in neoplastic cells, which results in worse prognosis and shorter survival time for patients $(4,14)$.

Determination of molecular subtypes and their combination with histopathological subtypes will give the clinic more factual information. In our study, molecular subgroups were determined with the immunohistochemical panel. However, due to the lack of technological possibilities, the incapacity to look at transcription profiles or whole gene analysis with NGS is an important limitation of our study. Like other CNS tumors, better and more detailed identification of genetic mutations in medulloblastoma will aid in the development of targeted therapies and prolong survival.

\section{ACKNOWLEDGEMENTS}

This study was approved by Baskent Universty Institutional Review Board (Project No: KA18/311) and supported by Baskent Universty Research Fund.

\section{REFERENCES}

1. Babaoglu B: Medulloblastom subtiplendirmesinde immünohistokimyasal yaklaşım (Uzmanlık Tezi), Ankara: Hacettepe University, 2017:1-73

2. Cambruzzi E: Medulloblastoma, WNT-activated/SHH-activated: Clinical impact of molecular analysis and histogenetic evaluation. Childs Nerv Syst 34:809-815, 2018

3. Craveiro RB, Ehrhardt M, Velz J, Olschewski M, Goetz B, Pietsch T, Dillao D: The anti-neoplastic activity of Vandetanib against high-risk medulloblastoma variants is profoundly enhanced by additional P13K inhibition. Oncotarget 8: 4691546927, 2017

4. David NL, Hiroko O, Otmar DW: WHO Classification of Tumours of the Central Nervous System. Revised $4^{\text {th }}$ ed. Lyon, 2016:183-200

5. Ellison DW, Dalton J, Kocak M, Nicholson SL, Fraga C, Neale G, Kenney A, Brat DJ, Perry A, Yong WH, Taylor RE, Bailey S, Clifford SC, Gilbertson RJ: Medulloblastoma: Clinicopathological correlates of $\mathrm{SHH}$, WNT, and $\mathrm{NonSHH} /$ WNT molecular subgroups. Acta Neuropathologica 121:381396, 2011 
6. Gibson P, Tong Y, Robinson G, Thompson MC, Currle DS, Eden C, Kranenburg TA, Hogg T, Poppleton H, Martin J, Finkelstein D, Pounds S, Weiss A, Patay Z, Scoggins M, Ogg R, Pei Y, Yang ZJ, Brun S, Lee Y, Zindy F, Lindsey JC, Taketo MM, Boop FA, Sanford RA, Gajjar A, Clifford SC, Roussel MF, McKinnon PJ, Gutmann DH, Ellison DW, Gilbertson R: Subtypes of medulloblastoma have distinct developmental origins. Nature 468:1065-1069, 2010

7. Goschzik T, ZurMühlen A, Kristiansen G, Haberler C, Stefanits $\mathrm{H}$, Friedrich C, Hoff K, Rutskowski S, Pfister SM, Pietsch T: Molecular stratification of medulloblastoma: Comparison of histological and genetic methods to detect Wnt activated tumours. Neuropathol Appl Neurobiol 41:135-144, 2015

8. Gottardo NG, Gajjar A: Current therapy for medulloblastoma. Curr Treat Options Neurol 8:319-334, 2006

9. Haberler C, Laggner U, Slavc I, Czech T, Ambros IM, Ambros PF, Budka H, Hainfellner JA: Immunohistochemical analysis of ini1 protein in malignant pediatric cns tumors: Lack of INI1 in atypical teratoid/rhabdoid tumors and in a fraction of primitive neuroectodermal tumors without rhabdoid phenotype. Am J Surg Pathol 30:1462-68, 2006

10. Herrlinger U, Steinbrecher A, Rieger J, Hau P, Kortmann RD: Adult medulloblastoma: Prognostic factors and response to therapy at diagnosis and at relapse. J Neurol 252:291-299, 2005

11. Hollmann TJ, Hornick JL: INI1-deficient tumors: Diagnostic features and molecular genetics. Am J Surg Pathol 35:47-63, 2011

12. Kool M, Korshunov A, Remke M, Jones DT, Schlanstein M, Northcott PA, Cho, YJ, Koster J, Meeteren AS, Vuurden D, Clifford SC, Pietsch T, Bueren A, Rutkowski S, McCabe M, Collins V, Haberler C, Bourdeaut F, Delattre O, Doz F, Ellison DW, Gilbertson R, Pomeroy S, Taylor M, Lichter P, Pfister S: Molecular subgroups of medulloblastoma: An international meta-analysis of transcriptome, genetic aberrations, and clinical data of WNT, SHH, Group 3, and Group 4 medulloblastomas. Acta Neuropathologica 123:473-484, 2012

13. Louis DN, Perry A, Burger P, Ellison DW, Reifenberger G, Deimling AV, Aldape K, Brat D, Collins VP, Eberhart C, FigarellaBranger D, Fuller GN, Giangaspero F, Giannini C, Hawkins C, Kleihues P, Korshunov A, Kros JM, Beatriz Lopes M, Ng HK, Ohgaki H, Paulus W, Pietsch T, Rosenblum M, Rushing E, Soylemezoglu F, Wiestler O, Wesseling P: International Society Of Neuropathology-Haarlem International Society of neuro pathology-Haarlem consensus guidelines for nervous system tumor classification and grading. Brain Pathol 24: 429-435, 2014

14. Mantovani F, Collavin L, Del Sal G: Mutant p53 as a guardian of the cancer cell. Cell Death Differentiation 26:199-212, 2019

15. Marshall GM, Carter DR, Cheung BB, Liu T, Mateos MK, Meyerowitz JG, Weiss WA: The prenatal origins of cancer. Nat Rev Cancer 14:277-289, 2014
16. Northcott PA, Korshunov A, Witt H, Hielscher T, Eberhart CG, Mack S, Bouffet E, Clifford SC, Hawkins CE, French P, Rutka JT, Pfister S, Taylor MD: Medulloblastoma comprises four distinct molecular variants. J Clin Oncol 29:1408-1414, 2010

17. Northcott PA, Shih DJ, Remke M, Kool M, Hawkins C, Eberhart CG, Dubuc A, Guettouche T, Cardentey Y, Bouffet E, Pomeray SL, Marra M, Malkin D, Rutka JT, Korshunov A, Pfister S, Taylor MD: Rapid, reliable, and reproducible molecular sub-grouping of clinical medulloblastoma samples. Acta Neuropathologica 123:615-626, 2012

18. Orr BA: Pathology, diagnostic, and classification of medulloblastoma. Brain Pathol 30:664-678, 2020

19. Paulino AC: Current multimodality management of medulloblastoma. Curr Probl Cancer 26:317-356, 2002

20. Pietsch T, Haberler C: Update on the integrated histopathological and genetic classification of medulloblastoma a practical diagnostic guideline. Clin Neuropathol 35:344-352, 2016

21. Ramaswamy V, Nör C, Taylor MD: P53 and medulloblastoma. Cold Spring Harb Perspect Med 6:1-8, 2016

22. Wu KS, Ho DM, Jou ST, Yu AL, Tran HM, Liang ML, Chen $\mathrm{H}$, Lee Y, Chen YW, Lin SC, Chang FC, Tsai ML, Liu YL, Lee HL, Hsieh K, Huang WC, Sung SY, Chang CC, Changou CA, Liang $\mathrm{KH}$, Hsieh TH, Liu YR, Wong TT: Molecular-clinical correlation in pediatric medulloblastoma: A cohort series study of 52 cases in Taiwan. Cancers (Basel) 12:653-677, 2020

23. Yu J, Zhao R, Shi W, Li H: Risk factors for the prognosis of pediatric medulloblastoma: A retrospective analysis of 40 cases. Clinics (Sao Paulo) 72:294-304, 2017

24. Zeltzer PM, Boyett JM, Finlay JL, Albright AL, Rorke LB, Milstein JL, Allen JC, Stevens KR, Stanley P, Li H, Wisoff JH, Geyer JR, Cullen P, Stehbens JA, Shurin SB, Packer RJ: Metastasis stage, adjuvant treatment, and residual tumor are prognostic factors for medulloblastoma in children: Conclusions from the Children's Cancer Group 921 randomized phase III study. J Clin Oncol 17:832-845, 1999

25. Zhang ZY, Xu J, Ren Y, Wai Li KK, Keung Ng H, Mao Y, Zhong P, Yao Y, Zhou LF: Medulloblastoma in China: Clinicopathologic analyses of SHH, WNT, and Non-SHH/WNT molecular subgroups reveal different therapeutic responses to adjuvant chemotherapy. PLoS ONE 16:1-8, 2014

26. Zhukova N, Ramaswamy V, Remke M, Pfaff E, Shih DJ, Martin DC, Branco PC, Baskin B, Ray P, Bouffet E, Bueren A, Jones D, Northcott PA, Kool M, Sturm D, Pugh TJ, Pomeroy SL, Cho YJ, Pietsch T, Gessi M, Rutkowski S, Bognar L, Klekner A, Cho BK, Kim SK, Wang KC, Eberhart CG, Fevre-Montange M, Fouladi M, French PJ, Kros M, Grajkowska WA, Gupta N, Weiss WA, Hauser P, Jabado N, Jouvet A, Jung S, Kumabe T, Lach B, Leonard JR, Rubin JB, Liau LM, Lassimi L, Pollack IF, Zitterbart K, Schller U, Hill RM, Lindsey JC, Bailey S, Ellison DW, Hawkin C: Subgroup-specific prognostic implications of TP53 mutation in medulloblastoma. J Clin Oncol 31:29272935, 2013 